\title{
Analysis of Risk Management for the Coal Mine Operations
}

\author{
Yaqian $\mathrm{Qi}^{1}$, Tong $\mathrm{Xu}^{2}$, Jun Steed Huang ${ }^{*}$ \\ ${ }^{1}$ Hohai University, Nanjing, China \\ ${ }^{2}$ Jiangsu University, Zhenjiang, China \\ ${ }^{3}$ Suqian College, Suqian, China \\ Email: *steedhuang@ujs.edu.cn
}

How to cite this paper: Qi, Y.Q., Xu, T. and Huang, J.S. (2017) Analysis of Risk Management for the Coal Mine Operations. Energy and Power Engineering, 9, 12-18. https://doi.org/10.4236/epe.2017.94B002

Received: November 4, 2016

Accepted: March 30, 2017

Published: April 6, 2017

\begin{abstract}
More than half of the energy sources in China are provided by combustion of coal from thermal power stations. By lowering the risk of excavating coal mines, we can decrease the price of the energy. This paper stresses the danger of coal mine gas explosions and constructs a gas explosion risk prediction system based on standard Hypo-Variance by using Brain Storms Optimization implemented with MATLAB simulation software. Hypo-Variance is the new concept built on the basis of Gauss Variance and can be used to make more precise risk assessments. By analyzing the multiple connections among gas density, temperature, humidity, pressure, air velocity, we can predict the trend of the gas event and propose the most safely timed schedule: operating three shifts in a day starting from a particular hour right after the gas peaking is over.
\end{abstract}

\section{Keywords}

Hypo-Variance, Gauss Distribution, Coal, Mine, Hazard

\section{Introduction}

According to the analysis of Jipin Sun [1], from China Mine University, in terms of mine accidentcategory, the death rate and national mine accident rate: roof $52.7 \%$, and $36.8 \%$, gas $11.3 \%$ and $29.7 \%$, transportation: $16.9 \%$ and $11.3 \%$, water disaster $3.1 \%$ and $8.1 \%$, electrical-mechanic $4.2 \%$ and $2.5 \%$, machine boom $2.7 \%$ and $1.9 \%$, fire disaster $0.4 \%$ and $1.9 \%$ from 2004 to 2013 . The water and fire accidents caused high death rate, others reversal. We think the high proportion is due to the unpredictable terrain. Compared to gas, water, fire accidents, transportation, machine boom accidents are easily avoidable if we operate properly. In theory, the distribution of gas and water is fractal. In other words, no matter how short of an interval of drilling the gas releasing hole, there is a possibility that we can miss an origin of gas fire. In order to avoid unnecessary loss, it is vi- 
tal to predict gas peaking time and the location of the gas leakage.

\section{Design Theory and Process}

\subsection{Design Theory}

1) Acquisition parameters:

Positive pressure: converted from negative pressure of drainage

Temperature: air temperature of drainage pipeline

Gas level: instantaneous concentration of gas in drainage pipeline

- Working flow: instantaneous flow rate* flow coefficient

2) The explanation of the formulae:

a) Pearson Product-moment Correlation Coefficient: PPCC, describes the linear correlation of two random variables. Karl Pearson put this forward in the 1880 s [2]. It is widely accepted in many aspects of science.

The definition of the coefficient:

$$
R=\frac{\sum_{i=1}^{n}\left(x_{i}-\bar{x}\right)\left(y_{i}-\bar{y}\right)}{\sqrt{\sum_{i=1}^{n}\left(x_{i}-\bar{x}\right)^{2} \sum_{i=1}^{n}\left(y_{i}-\bar{y}\right)^{2}}}
$$

where the subscribe $i$ runs from 1 to $\mathrm{n}, \mathrm{n}$ is the number of terms.

b) Hypo-(or Huang) Product-moment Correlation Coefficient: HPCC, measures the nonlinear correlation of two variables. It was put forward by Prof. Steed Huang in 2015 [3].

The definition of Correlation Coefficient

$$
R s=\frac{\left(\sum_{i=1}^{n}\left(x_{i}-\bar{x}\right)^{\frac{3}{4}}\left(y_{i}-\bar{y}\right)^{\frac{3}{4}}\right)^{\frac{4}{3}}}{\left(\sum_{i=1}^{n}\left(x_{i}-\bar{x}\right)^{\frac{3}{2}} \sum_{i=1}^{n}\left(y_{i}-\bar{y}\right)^{\frac{3}{2}}\right)^{\frac{2}{3}}}
$$

Huang Product-moment Correlation Coefficient is used to measure the nonlinear correlation of three variables. It can be generalized from the form of two variables to three and can be used in many aspects of industry.

c) Three Factor [4] Correlation Coefficient (TFCC) definition:

$$
R s=\frac{\left(\sum_{i=1}^{n}\left(x_{i}-\bar{x}\right)^{\frac{1}{2}}\left(y_{i}-\bar{y}\right)^{\frac{1}{2}}\left(z_{i}-\bar{z}\right)^{\frac{1}{2}}\right)^{2}}{\left(\sum_{i=1}^{n}\left(x_{i}-\bar{x}\right)^{\frac{3}{2}} \sum_{i=1}^{n}\left(y_{i}-\bar{y}\right)^{\frac{3}{2}} \sum_{i=1}^{n}\left(z_{i}-\bar{z}\right)^{\frac{3}{2}}\right)^{\frac{2}{3}}}
$$

d) Multiple Factor [5] Correlation Coefficient: If we want to analyze the correlation of four variables, density, temperature, pressure, flow velocity, of gas, then we should define the Multiple Factors Correlation Coefficient (MFCC):

$$
R s=\frac{\left(\sum_{i=1}^{n} \prod_{j=2}^{N}\left(x_{i, j}-\bar{x}\right)^{\frac{3}{2 N}}\right)^{\frac{2 N}{3}}}{\left(\prod_{j=2}^{N} \sum_{i=1}^{n}\left(x_{i, j}-\bar{x}\right)^{\frac{3}{2}}\right)^{\frac{2}{3}}}
$$


where the subscribe i runs from 1 to $n, n$ is the number of terms, the subscribe $j$ runs from 2 to $N, N$ is the number of variables. It is similar to the Fractional Variance [6] defined by the Gamma function.

3) Formula comparison

a) The difference and advantage of Huang Product-moment Correlation Coefficient compared with Pearson Product-moment Correlation Coefficient: by using the 1.5 times instead of square, the new definition can reflect the fluctuating asymmetry, fitting the nature of things more accurately and can be used for multiple factors in more aspects of the fields.

b) The difference, disadvantage and advantage by using field between Hypo Product-moment Correlation Coefficient: The new definition, not square, but with 1.5 parties, can reflect the asymmetry of fluctuation and can be extended to the occasion of multiple targets; the disadvantage is that the calculation is relatively complex. If the variable is strictly consistent with the Gauss distribution and the fluctuation is statistically symmetric or only involves two target variables, it is not necessary to use the new definition.

4) The Brain Storm Optimization algorithm

Brain Storm Optimization (BSO) algorithm [7] and then correlation criterion of gas outburst prediction orientation curve, its principle diagram is as follows in Figure 1.

\subsection{Maintaining the Integrity of the Specifications}

1) Calculation methods and steps

According to the gas concentration, we treat each pumping station as a set of data units, the ground station data as a high concentration data group, and the

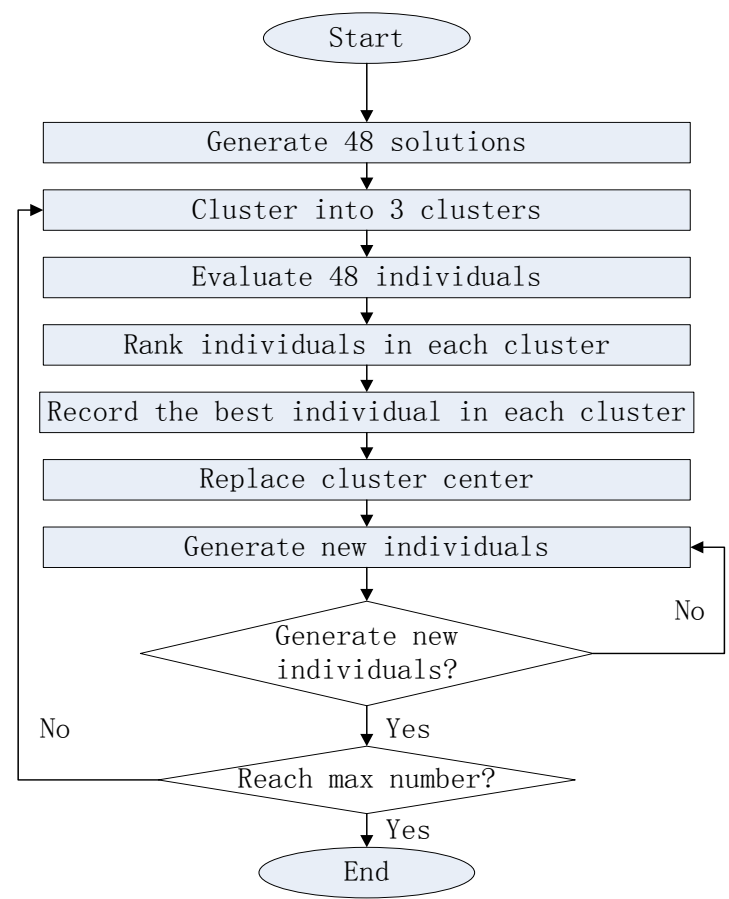

Figure 1. Example of brain storming optimization algorithm. 
mobile station data as a low concentration of data.

Then take the entire mining area of low or high concentrations average as a benchmark of respected concentration average for optimization, searching for the difference between the low and high concentration with optimized correlation coefficient, thus inversion derivation and deduction of geological characteristics of coal and gas outburst extrusioncurve.

Assume that the mine space is closed, or said each ventilation are all under observation, it has the integrity of the data, or said outburst only through the monitoring of air discharge, no other unseen conduits, for us to meet the law of conservation for the temperature, pressure, velocity and concentration.

2) Pattern analysis

We found that the gas outburst is cyclical, at about every two to three climaxes a day, estimation that is related to the earth core has the eccentric phase rotary extrusion towards the crust of our planet.

The next task is to establish the monthly benchmark data. According to the data on that day and of input variables: gas concentration. We use brainstorm to optimize and look for the cycle time of today to predict the trends for tomorrow.

The topology of the ground station and the mobile station is needed to calculate the correlation coefficient of the location, and using the position, to predict the periodicity of the space pattern and predict the exact danger area.

\section{Design Procedures and Results}

\subsection{Program Instructions}

a(day).xls is the original data contains day 15 to day 31 .

a15-a31 compressed package is the original data (standby)

BsoMain.m is the main program and brain storm subroutine

CfdMg.m is a complex number of variance criterion subroutine

chuli.m is the three correlation coefficient calculation subroutine

- function $\mathrm{z}=\operatorname{CfdMg}(\mathrm{x})$

- $\mathrm{n}=$ length $(\mathrm{x})-1$;

- $\mathrm{z}=0$;

- global x24

- $\mathrm{x} 24=\operatorname{round}\left(\mathrm{x}^{\star} 5\right)$;

- gasHig= xlsread('date.xls','a1:x1');

- gasLow=xlsread('date.xls','a2:x2);

- $\quad$ THig= xlsread('date.xls','a3:x3')';

- $\quad$ TLow = xlsread('date.xls','a4:x4')';

- $\quad$ PHig= xlsread('date.xls','a5:x5')';

- $\quad$ PLow = xlsread('date.xls','a5:x5')';

- $\operatorname{gasHig}=$ xlsread('date.xls','a6:x6')';

- $\mathrm{THigH}=\mathrm{THig}{ }^{\star} 1.8+32^{\star}$ ones $(24,1)$;

- $\mathrm{TLowH}=\mathrm{TLow}^{\star} 1.8+32^{\star}$ ones $(24,1)$;

- $\quad$ HHigZ $=101.325^{\star}$ ones(24,1)-PHig;

- $P L o w Z=101.325^{\star}$ ones(24,1)-PLow; 
- $\quad$ energH=gasHig $+\mathrm{x} 24$;

- energL=gasLow;

- $\mathrm{z}=\mathrm{abs}$ (chuli(energH,THigH,PHigZ)-chuli(energL,TLowH,PLowZ));

More of the related code is available for download at the MATLAB server site.

\subsection{Calculation Results}

We found that the gas concentration changes periodically. There are about three peaks every day due to the estimation of the earth's core and the eccentric rotary phase extrusion relative to the earth crust. The first picture of Figure 2 shows the calculation results of the distance between each round of the brainstorming algorithm and the final optimization goal. The blue (high) curve in the second picture of Figure 3 is our predictive value for the data. The red (low) curve is the

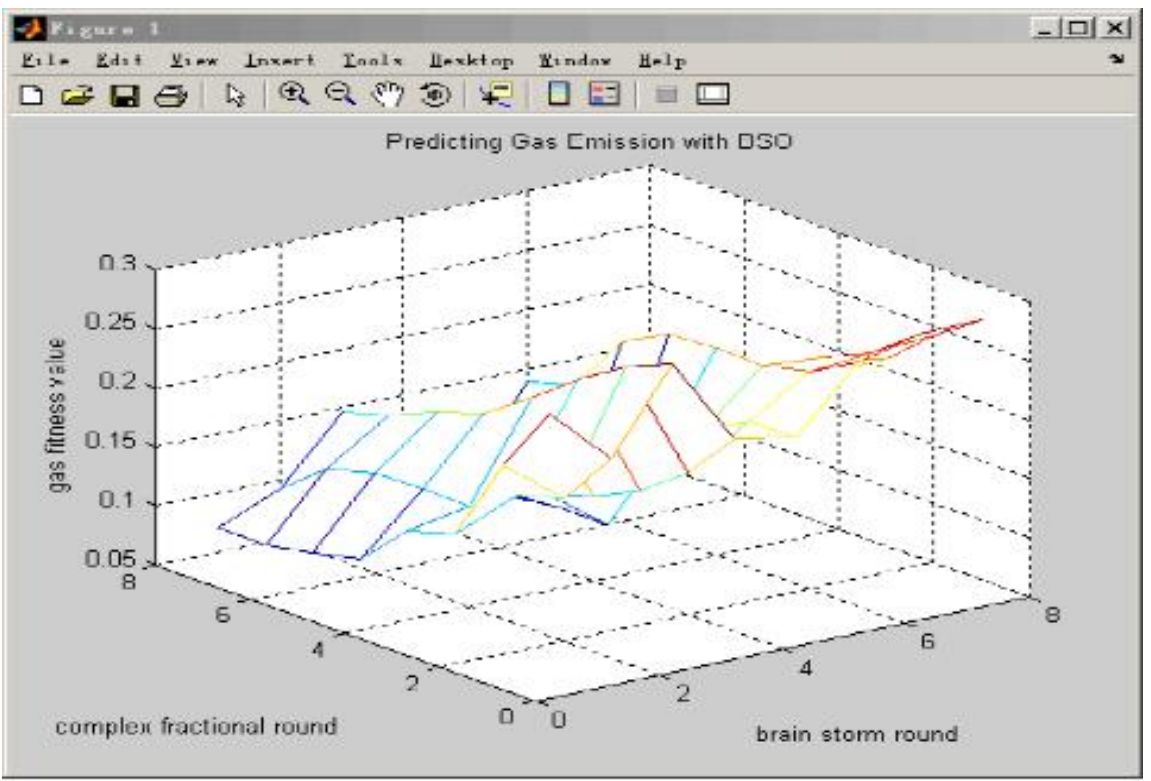

Figure 2. Example of brain storming optimization fitness.

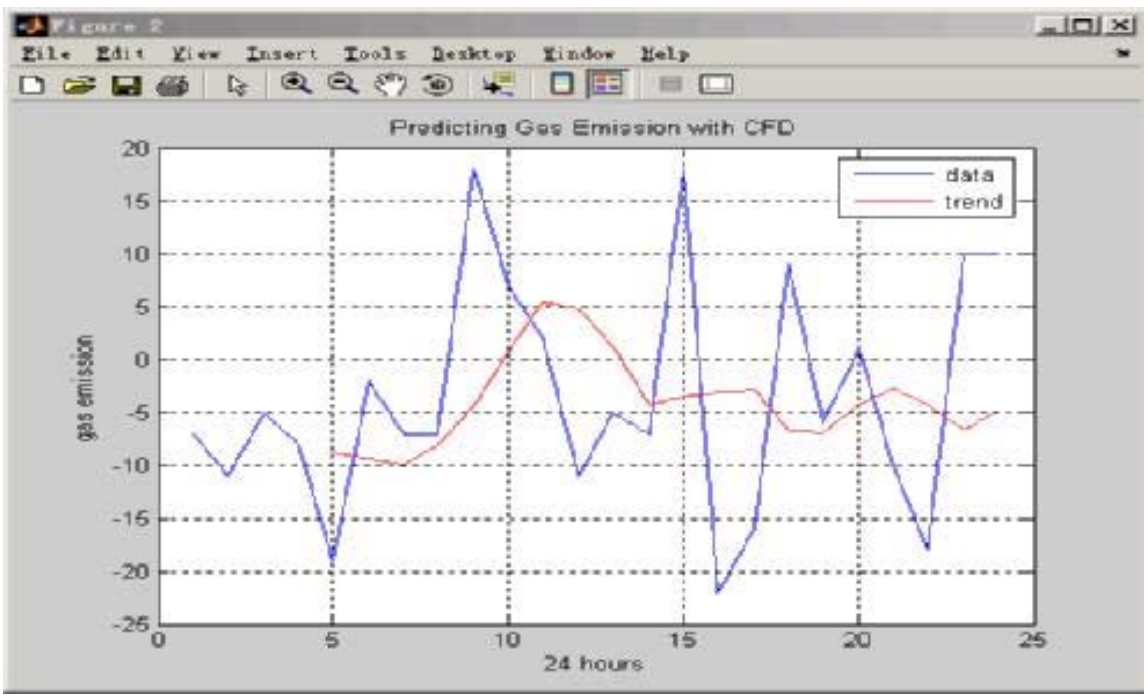

Figure 3. Example of predicted gas emission cycle. 
trend curve after the average value is taken every four hours.

\section{Conclusions}

This experiment was achieved by performing simulation though the Matlab software [8]. It is more convenient to use the MATLAB software to calculate the fractional variance but in principle, any other scientific computing software can achieve a similar function. The purpose of this study is through the prediction of mine gas using the MATLAB software, we can collect the temperature, pressure, ground station and mobile station of gas flow in the mines for the analysis of correlation, correlation coefficient, and using the brains-storm algorithm to optimize the prediction of the gas emission peaking and the future trend.

Some difficulties are: the algorithm chuli.m changes for ternary correlation coefficient algorithm from binary case, and in CfdMg.m function when modify initial value, call the function parameters, using a formula to turn in between Fahrenheit and Celsius, or the negative pressure is getting to positive pressure. We finally observed in graphics, estimated daily high gas concentration duration of peak hours. We recommend 8 hours a shift so that miners will be safe and can rest during the high gas emission period and return to work on a low gas time period so that the explosion risk is reduced to the lowest level.

\section{Acknowledgements}

Thanks to Professor Yuhui Shi from SUST for providing the brain storming algorithm plus subroutines, thanks to Mr. Zhao and Mr. Ding from China Coal provide the coal mine data sets, and thanks to Ms. Sun Chenxu and 98 Suqian students to assist us in completing the original data calculation and verification for the 25 working days records, that gives us the confidence for seeing the same pattern, finally, thanks to Kenneth Huang from UCSD for editing the English writing.

\section{References}

[1] Sun, J.P. (2010) Coal Mine Safety Production Monitoring and Communication Technology. Journal of China Coal Society, 135.

[2] Pearson, K. (1895) Notes on Regression and Inheritance in the Case of Two Parents Proceedings of the Royal Society of London, 58, 240-242. https://doi.org/10.1098/rspl.1895.0041

[3] Zou, Q., Hu, Y.F. and Huang, J.S. (2015) Definition of Complex Hurst and Fractional Analysis for Stock Market Fluctuation , 2nd International Conference on Industrial Engineering, Management Science and Applications.

[4] Braun, T., Rich, M. and Kramer, M.F. (2013) Correlation of Three Variables Describing Nasal Patency (HD, MCA, NOSE Score) in Healthy Subjects. Brazilian Journal of Otorhinolaryngology, 79, 354-358. https://doi.org/10.5935/1808-8694.20130062

[5] Timothy, K. (2006) Multiple Regression and Beyond. Boston: Pearson Education.

[6] Hazewinkel and Michiel (2001) Gamma Function. Encyclopedia of Mathematics. 
[7] Shi, Y.H. (2011) An Optimization Algorithm Based on Brainstorming Process. International Journal of Swarm Intelligence Research, 2.

[8] Martinez, W.L. and Martinez, A.R. (2016) Computational Statistics Handbook with MATLAB. 3rd Edition, Chapman \& Hall/CRC.

Submit or recommend next manuscript to SCIRP and we will provide best service for you:

Accepting pre-submission inquiries through Email, Facebook, LinkedIn, Twitter, etc. A wide selection of journals (inclusive of 9 subjects, more than 200 journals) Providing 24-hour high-quality service User-friendly online submission system Fair and swift peer-review system Efficient typesetting and proofreading procedure Display of the result of downloads and visits, as well as the number of cited articles Maximum dissemination of your research work

Submit your manuscript at: http://papersubmission.scirp.org/ Or contact epe@scirp.org 